\title{
ON THE DIAPHONY OF ONE CLASS OF ONE-DIMENSIONAL SEQUENCES
}

\author{
VASSIL ST. GROZDANOV
}

Department of Mathematics

University of Blagoevgrad

2700 Blagoevgrad, Bulgaria

(Received November 7, 1991 and in revised form August 15, 1992)

\begin{abstract}
In the present paper, we consider a problem of distribution of sequences in the interval $[0,1)$, the so-called ' $P_{r}$-sequences' We obtain the best possible order $O\left(N^{-1}(\log N)^{1 / 2}\right)$ for the diaphony of such $P_{r}$-sequences For the symmetric sequences obtained by symmetrization of $P_{r}$ sequences, we get also the best possible order $O\left(N^{-1}(\log N)^{1 / 2}\right)$ of the quadratic discrepancy
\end{abstract}

KEY WORDS AND PHRASES Distribution of sequences, quadratic discrepancy and Pr-sequences 1991 AMS SUBJECT CLASSIFICATION CODE $11 \mathrm{~B} 83$

\section{INTRODUCTION}

11 Let $\sigma=\left(x_{n}\right)_{n=0}^{\infty}$ be an infinite sequence in the unit interval $E=[0,1)$ For every real number $x \in E$ and every positive integer $N$ we denote $A_{N}(\sigma, x)$ the number of terms $x_{n}, 0 \leq n \leq N-1$, which are less than $x$

The sequence $\sigma$ is called uniformly distributed in $E$ if for every real number $x \in E$ we have

$$
\lim _{N \rightarrow \infty} A_{N}(\sigma ; x) N^{-1}=x .
$$

The systematic study of the theory of uniformly distributed sequences was initiated by Weyl [1]

A classical measure for the irregularity of the distribution of a sequence $\sigma$ in $E$ is its quadratic discrepancy $T_{N}(\sigma)$, which is defined for every positive integer $N$ as

$$
T_{N}(\sigma)=\left(\int_{0}^{1}\left|A_{N}(\sigma ; x) / N-x\right|^{2} d x\right)^{1 / 2} .
$$

The irregularity of distribution with respect to the quadratic discrepancy was first studied by Roth [2]

In 1976, Zinterhof (see [3,4]) proposed a new measure for distribution, which he named diaphony The diaphony $F_{N}(\sigma)$ of $\sigma$ is defined for every positive integer $N$ as

$$
F_{N}(\sigma)=\left(2 \sum_{h=1}^{\infty} h^{-2}\left|N^{-1} S_{N}(\sigma ; h)\right|^{2}\right)^{1 / 2}
$$

where

$$
S_{N}(\sigma ; h)=\sum_{n=0}^{N-1} \exp \left(2 \pi i h x_{n}\right)
$$

signify trigonometric sum of $\sigma$

We note that the diaphony of $\sigma$ can be written in the form

$$
F_{N}(\sigma)=\left(N^{-2} \sum_{n, k=0}^{N-1} g\left(x_{n}-x_{k}\right)\right)^{1 / 2}
$$

where

$$
g(x)=\pi^{2}\left(2 x^{2}-2 x+1 / 3\right)
$$

It is well known (see [5], p 115, [4]) that both equalities

$$
\lim _{N \rightarrow \infty} T_{N}(\sigma)=0 \text { and } \lim _{N \rightarrow \infty} F_{N}(\sigma)=0
$$

are equivalent to the definition that the sequence $\sigma$ is uniformly distributed in $E$ 
12 Using the well-known theorem of Roth [2] it can be proved (see Neiderreiter [7], p 158; Proinov [8]) that for any infinite sequence $\sigma$ in $E$, the estimate

$$
T_{N}(\sigma)>214^{-1} N^{-1}(\log N)^{1 / 2}
$$

holds for infinitely many integers $N$ The exactness of the order of magnitude of this estimate was proved by Proinov ([9], [10], [11])

Proinov [8] proved that for any sequence $\sigma$ in $E$ the estimate

$$
F_{N}(\sigma)>68^{-1} N^{-1}(\log N)^{1 / 2}
$$

holds for infinitely many $N$.

From (1.1) and (1.2) becomes clearly that the best possible order of diaphony and quadratic discrepancy of every sequence $\sigma$ in $E$ is $O\left(N^{-1}(\log N)^{1 / 2}\right)$.

2. A SEQUENCE OF $r$-ADIC RATIONAL TYPE.

\subsection{CONSTRUCTION OF SEQUENCE OF $r$-ADIC RATIONAL TYPE}

In this part we generalize Sobol's ([12], [5], p 117, [13], p. 23) construction of sequences of binary rational type

Let $r \geq 2$ is fixed integer. We consider the infinite matrix

$$
\left(v_{s, j}\right)=\left(\begin{array}{ccc}
v_{11} & v_{21} & --- \\
v_{12} & v_{22} & --- \\
--- & --- & ---
\end{array}\right)
$$

where for every $s, j=1,2, \cdots, v_{s, j} \in\{0,1, \cdots, r-1\}$. We suppose that in every column, the quantity of $v_{s, j}$, which are different from zero is a positive integer number, i.e., $v_{s, j}=0$ for $j$ sufficiently big Such matrix we shall call guiding matrix

To every column of the matrix $(21)$ corresponds a $r$-adic rational numbers

$$
V_{s}=0, v_{s, 1} v_{s, 2} \cdots v_{s, j} \cdots(s=1,2 \cdots)
$$

The numbers determined in (2.2) are called guiding numbers

We signify $N_{0}=N \cup\{0\}$, with $N$ the set of natural integers.

A sequence of $r$-adic rational type (or $R P$-sequence) is a sequence $(\varphi(i))_{\imath=0}^{\infty}$, which is generated by the guiding matrix $\left(v_{s, j}\right)$ in the following way: If in the $r$-adic number system

$$
i=e_{m} e_{m-1} \cdots e_{1}
$$

then in the $r$-adic number system

where for $j=1,2, \cdots, m$

$$
\varphi(i)=0, W_{1}^{*} W_{2}^{*} \ldots{ }^{*} W_{m}
$$

$$
W_{\jmath}=e_{\jmath} V_{\jmath}=\underbrace{V_{j}^{*} V_{j}^{*} \cdots V^{*}}_{e_{\jmath}-\text { terms }},
$$

and * is the operation of the digit-by-digit addition modulo $r$ of elements of $Z_{r}=\{0,1, \cdots, r-1\}$.

A $R P$-sequence $(\varphi(i))_{\imath=0}^{\infty}$, which is generated by the guiding matrix $\left(v_{s, j}\right)$ can be also constructed by following the three mentioned below rules

(1) $\varphi(0)=0$.

(2) If $i=r^{s}\left(s \in N_{0}\right)$, then $\varphi(i)=V_{s+1}$.

(3) If $r^{s}<i<r^{s+1}$, then $\varphi(i)=e_{s+1} \varphi\left(r^{s}\right)^{*} \varphi\left(i-e_{s+1} r^{s}\right)$, where $e_{s+1}$ is higher significant digit in $r$-adic development of $i$ and $e_{s+1} \varphi\left(r^{s}\right)=\underbrace{V_{s+1}^{*} V_{s+1}^{*} \cdots V_{s+1}}_{e_{s+1}-\text { terms }}$.

Obviously the operation * has commutative and associative property.

We shall prove that the two definitions of the $P R$-sequences are equivalent.

Let us suppose that the first definition is valid for $R P$-sequence.

(1) If $i=0$, then obviously $\varphi(i)=0$.

(2) If $i=r^{s}\left(s \in N_{0}\right)$, then $\varphi(i)=V_{s+1}$. 
(3) Let us assume that $r^{s}<i<r^{s+1}$ and $i=\left(e_{s+1} e_{s} \cdots e_{1}\right)_{r}$. Since the operator * is commutative and associative we have

$$
\varphi(i)=0,\left(\left(e_{1} V_{1}\right)^{*} \ldots{ }^{*}\left(e_{s} V_{s}\right)\right)^{*}\left(e_{s+1} V_{s+1}\right) .
$$

Since $V_{s+1}=\varphi\left(r^{s}\right)$ and $i-e_{s+1} r^{s}=\left(e_{s} e_{s-1} \cdots e_{1}\right)_{r}$, then $\varphi\left(i-e_{s+1} r^{s}\right)=0,\left(e_{1} V_{1}\right)^{*} \ldots{ }^{*}\left(e_{s}\right.$ $\left.V_{s}\right)$. Finally $\varphi(i)=e_{s+1} \varphi\left(r^{s}\right)^{*} \varphi\left(i-e_{s+1} r^{s}\right)$. The three rules in the second definition for $R P$ sequence are proved.

Reversely, let the second definition for $P R$-sequence is valid and $i$ is given positive integer. Then there exists uniquely positive integer $s$ that $r^{s} \leq i<r^{s+1}$. We shall prove definition 1 by induction on $s$. If $s=0$, then $1 \leq i<r$ and

$$
\varphi(i)=i \varphi\left(r^{0}\right)^{*} \varphi(0)=0, i V_{1} .
$$

We make inductive supposition that for some $s \in N$ and every integer $i, r^{s-1} \leq i<r^{s}$ definition 1 holds. Let us assume that $r^{s} \leq i<r^{s+1}$ and $i=\left(e_{s+1} e_{s} \cdots e_{1}\right)_{r}$. From rule 3 we have

$$
\varphi(i)=e_{s+1} \varphi\left(r^{s}\right)^{*} \varphi\left(i-e_{s+1} r^{s}\right) .
$$

If we denote $j=i-e_{s+1} r^{s}$, then $j=\left(e_{s} e_{s-1} \cdots e_{1}\right)_{r}$ and $r^{s-1} \leq j<r^{s}$. Then by inductive supposition

$$
\varphi(j)=0,\left(e_{1} V_{1}\right)^{*} \ldots{ }^{*}\left(e_{s} V_{s}\right) .
$$

By rule 2, $\varphi\left(r^{s}\right)=V_{s+1}$ and we have

$$
\varphi(i)=0,\left(e_{1} V_{1}\right)^{*} \ldots{ }^{*}\left(e_{s} V_{s}\right)^{*}\left(e_{s+1} V_{s+1}\right) .
$$

Definition 1 holds for every positive integer $s$.

In the following lemma we give a property of the functions $\varphi$.

LEMMA 2.1. Let $\left(v_{s, j}\right)$ is an arbitrary guiding matrix, and $(\varphi(i))_{i=0}^{\infty}$ is $R P$-sequence, which is generated by $\left(v_{s, j}\right)$. Let $\nu, m, n$ be integer numbers such that $\nu \in N_{0}, 0 \leq n<r^{\nu}$ and $m \equiv 0(\bmod$ $\left.r^{\nu}\right)$. Then we have

The proof of the lemma is obvious.

$$
\varphi(m+n)=\varphi(m)^{*} \varphi(n)
$$

For every integer $a \in Z_{r}$ we define $\bar{a}$ the only integer, which is a solution of the equation

$$
a+\bar{a} \equiv 0(\bmod r)
$$

If $\alpha=0, \alpha_{1} \alpha_{2} \cdots \alpha_{t}$, where, for $\tau=1,2, \cdots, t \quad \alpha_{\tau} \in Z_{r}$, then we define $\bar{\alpha}=0, \bar{\alpha}_{1} \bar{\alpha}_{2} \cdots \bar{\alpha}_{t}$.

\subsection{SEQUENCES OF $r$-ADIC RATIONAL TYPE, WHICH ARE $P_{r}$-SEQUENCES.}

The theory of the $P_{r}$-sequences was first studied by Faure $([14] ;[15])$ and generalized by Neiderreiter ([16];[17]).

A $r$-adic elementary interval is an interval

$$
l_{m, j}=\left[(j-1) / r^{m}, j / r^{m}\right),
$$

in which $1 \leq j \leq r^{m}$, for any integer $m$.

Let $N=r^{m}$. We shall call the net

$$
X=\left(x_{0}, x_{1}, \cdots, x_{N-1}\right)
$$

be a net of type $P_{r}^{m}$ (or $P_{r}^{m}$-type), if every $r$-adic elementary interval $l_{m, \jmath}$, having length $1 / N$ contain one point of the net $X$.

A $r$-adic section of the sequence $X=\left(x_{i}\right)_{\imath=0}^{\infty}$ is a set of terms $x_{\imath}$, with numbers $i$, satisfying the inequalities

$$
k r^{s} \leq i<(k+1) r^{s}
$$

for every integers $k$ and $s$, such that $k=0,1, \cdots ; s=1,2, \cdots$.

The sequence $\left(x_{\imath}\right)_{i=0}^{\infty}$ is called a sequence of type $P_{r}$ (or $P_{r}$-sequence) if every $r$-adic section is a $P_{r}^{m}$-net.

THEOREM 2.1. Let in the guiding matrix $\left(v_{s, j}\right)$ every $v_{s, s}=1$ and for $j>s$ every $v_{s, j}=0$, i.e., 


$$
\left(v_{s, \jmath}\right)=\left(\begin{array}{cccccc}
1 & v_{21} & v_{31} & --- & v_{\jmath 1} & -- \\
0 & 1 & v_{32} & --- & v_{\jmath 2} & -- \\
0 & 0 & 1 & --- & v_{\jmath 3} & -- \\
--- & --- & --- & --- & --- & --- \\
0 & 0 & 0 & --- & 1 & -- \\
--- & --- & --- & --- & --- & ---
\end{array}\right) .
$$

Then the corresponding $R P$-sequence is $P_{r}$-sequence

PROOF. We choose arbitrary $r$-adic section of the $R P$-sequence $(\varphi(i))_{\imath=0}^{\infty}$, the length of which is $r^{m}$. We write the numbers $i$, belonging to this section in the $r$-adic number system:

$$
i=c_{\mu} c_{\mu-1} \cdots c_{m+1} e_{m} e_{m-1} \cdots e_{1},
$$

where $c_{k}$ are fixed and $e_{k}$ are arbitrary $r$-adic numbers

We choose now an arbitrary $r$-adic interval $l$, with length $|l|=r^{-m}$. In the $r$-adic system this interval is determined by the inequality

$$
0, a_{1} a_{2} \cdots a_{m} \leq x<0, a_{1} a_{2} \cdots a_{m}+0, \underbrace{0 \cdots 0}_{m-\text { zeros }} 1,
$$

where $a_{1}, \cdots, a_{m}$ are $r$-adic numbers

We shall prove, that for every choice of the numbers $c_{k}$ and $a_{k}$ among the numbers $i$, in the form (2.4) there exists exactly one $i$, for which $\varphi(i) \in l$.

. In the $r$-adic number system we write

$$
\varphi(i)=0, g_{\imath, 1} g_{\imath, 2} \cdots g_{\imath, J} \cdots
$$

From (2.3) we have

$$
g_{\imath, J}=e_{1} v_{1, J}^{*} \ldots{ }^{*} e_{m} v_{m, j}^{*} c_{m+1} v_{m+1, J}^{*} \ldots{ }^{*} c_{\mu} v_{\mu, \jmath},
$$

where the sense of $e_{k} v_{k, j}$ is the same as in (2.3).

The condition $\varphi(i) \in l$ is equivalent to the following conditions

$$
g_{\imath, j}=a_{\jmath}, \text { for } 1 \leq j \leq m .
$$

We get that for each $j, 1 \leq j \leq m$

$$
g_{\imath, J}=\left(e_{1} v_{1, j}^{*} \ldots{ }^{*} e_{m} v_{m, J}\right)^{*}\left(c_{m+1} v_{m+1, J}^{*} \cdots{ }^{*} c_{\mu} v_{\mu, J}\right)
$$

from which we get

$$
e_{1} v_{1, \jmath}^{*} \cdots{ }^{*} e_{m} v_{m, \jmath}=a_{\jmath}^{*}\left(\overline{c_{m+1} v_{m+1, \jmath}^{*} \cdots{ }^{*} c_{\mu} v_{\mu, \jmath}}\right) \quad(1 \leq j \leq m)
$$

Let us call $f_{j}$ the right-side of (2.5) for $1 \leq j \leq m$. Having in mind that for $s=1,2, \cdots, v_{s, s}=1$ and in case $j>s, v_{s, j}=0$, the system (2.5) become

$$
e_{\jmath} v_{\jmath, \jmath}^{*} e_{\jmath+1} v_{\jmath+1, \jmath}^{*} \cdots{ }^{*} e_{m} v_{m, \jmath}=f_{\jmath}(1 \leq j \leq m)
$$

In this system the unknowns $e_{1}, e_{2}, \cdots, e_{m}$ are successively so determined that it has only one solution.

The theorem is proved.

In the following lemma we shall show some property of $P_{r}$-sequences.

LEMMA 2.2. Let $N=r^{\nu}$ where $\nu \in N_{0}$. For every guiding matrix $\left(v_{s, j}\right)$ in which $v_{s, s}=1$ and $v_{s, j}=0$ for $j>s(s=1,2, \cdots)$ and for the $R P$-sequence $(\varphi(i))_{\imath=0}^{\infty}$, which is product of $\left(v_{s, j}\right)$ we have

$$
\left\{\varphi(i): 0 \leq i<r^{\nu}\right\}=\{j / N: 0 \leq j<N\}
$$

PROOF. We shall make the proof by induction on $\nu$. If $\nu=0$ and $\nu=1$, then we make directly examination.

We make inductive supposition, that for some $\nu \in N$ the equality (26) is true and for $j=0,1, \cdots, r-1$ we consider the multitudes $A_{j}=\left\{\varphi(i): j r^{\nu} \leq i<(j+1) r^{\nu}\right\}$. Then obviously

$$
A=\bigcup_{\jmath=0}^{r-1} A_{\jmath}
$$

where $A=\left\{\varphi(i): 0 \leq i<r^{\nu+1}\right\}$.

We consider that $j=0$. By the inductive supposition

$$
A_{0}=\left\{\varphi(i): 0 \leq i<r^{\nu}\right\}=\left\{m / r^{\nu+1}: 0 \leq m<r^{\nu+1}, m \equiv 0(\bmod r)\right\}
$$


Let us now consider that $1 \leq j \leq r-1$. We shall prove the following equality.

$$
A_{\jmath}=\left\{m / r^{\nu+1}: 0 \leq m<r^{\nu+1}, m \equiv j(\bmod r)\right\} .
$$

Let $j, 1 \leq j \leq r-1$ is fixed integer and consider that $j r^{\nu} \leq i<(j+1) r^{\nu}$. Let us represent $i$ in the form $i=j r^{\nu}+k$, where $0 \leq k<r^{\nu}$.

Then by Lemma 21 we have

It is obvious that

$$
\varphi(i)=\varphi\left(j r^{\nu}\right)^{*} \varphi(k)
$$

Let us put

$$
\varphi\left(j r^{\nu}\right)=\underbrace{V_{\nu+1}^{*} V_{\nu+1}^{*} \cdots{ }^{*} V_{\nu+1}}_{j-\text { terms }}
$$

$$
\varphi\left(j r^{\nu}\right)=0, w_{\nu+1,1} w_{\nu+1,2} \cdots w_{\nu+1, \nu+1} .
$$

From (2 11) is clear, that $w_{\nu+1, \nu+1}=j$. Let $k$ has $r$-adic development $k=k_{\nu} k_{\nu-1} \cdots k_{1}$. Then

$$
\begin{gathered}
\varphi(k)=0,\left(k_{1} V_{1}\right)^{*} \cdots{ }^{*}\left(k_{\nu} V_{\nu}\right) \\
\left.\varphi(k)=0, a_{1} a_{2} \cdots a_{\nu}, \text { where } a_{s} \in\{0,1, \cdots, r-1\}, s=1,2, \cdots, \nu\right)
\end{gathered}
$$

From (2.10), (2.11) and (2.12) we get

$$
\varphi(i)=0,\left(a_{1}^{*} w_{\nu+1,1}\right) \cdots\left(a_{\nu}^{*} w_{\nu+1, \nu}\right) j=0, b_{1} b_{2} \cdots b_{\nu} j
$$

When $0 \leq k<r^{\nu}$, then $0 \leq\left(b_{1} b_{2} \cdots b_{\nu}\right)_{r}<r^{\nu}$ and from (2.13) we get that for $1 \leq j \leq r-1$

$$
A_{j}=\left\{\varphi(i): j r^{\nu} \leq i<(j+1) r^{\nu}\right\}=\left\{m / r^{\nu+1}: 0 \leq m<r^{\nu+1}, m \equiv j(\bmod r)\right\}
$$

The inequalities (2.9) are proved.

By induction on $\nu$ the lemma is proved

LEMMA 2.3 Let $(\varphi(i))_{\imath=0}^{\infty}$ be a $P_{r}$-sequence. Then for every $\nu \in N_{0}$ holds the equality

$$
\begin{aligned}
\{\varphi(m+j): m & \left.\equiv 0\left(\bmod r^{\nu}\right), 0 \leq j<r^{\nu}\right\} \\
& =\left\{\varphi(m)+\varphi(j)(\bmod 1): m \equiv 0\left(\bmod r^{\nu}\right), 0 \leq j<r^{\nu}\right\}
\end{aligned}
$$

PROOF. Let us consider that $m=k r^{\nu}$, for some positive integer $k$. The equality (2.14) is equivalent to the equality

$$
\begin{aligned}
\{\varphi(m+j): m & \left.\equiv 0\left(\bmod r^{\nu}\right), 0 \leq j<r^{\nu}\right\} \\
& =\bigcup_{l=0}^{r^{\nu-1}-1}\left\{\varphi(m+j): m \equiv 0\left(\bmod r^{\nu}\right), l r \leq j<(l+1) r\right\}
\end{aligned}
$$

First, we shall prove that for every fixed $l, 0 \leq l \leq r^{\nu-1}$ exists uniquely $l^{\prime} 0 \leq l^{\prime}<r^{\nu-1}$, such that

$$
\begin{aligned}
\{\varphi(m+j): m & \left.=k r^{\nu}, l r \leq j<(l+1) r\right\} \\
& =\left\{\varphi(m)+\varphi(j)(\bmod 1): m=k r^{\nu}, l^{\prime} r \leq j<\left(l^{\prime}+1\right) r\right\} .
\end{aligned}
$$

Let $k=\left(k_{n} k_{n-1} \cdots k_{1}\right)_{r}$. Then we have

$$
\varphi(m)=0, g_{1}^{m} g_{2}^{m} \cdot \cdots g_{n+\nu}^{m}
$$

where for $1 \leq i \leq n+\nu g_{2}^{m}=\sum_{h=1}^{n} k_{h} v_{h+\nu, i}(\bmod r)$.

Let $0 \leq l<r^{\nu-1}$ be fixed integer and $l=\left(l_{\nu-1} \cdots l_{1}\right)_{r}$. Then $l r=\left(l_{\nu-1} \cdots l_{1} 0\right)_{r}$.

When $j$ is such integer that $l r \leq j<(l+1) r$, we have $j=\left(l_{\nu-1} \cdots l_{1} l_{0}\right)_{r}$, where $l_{\nu-1}, \cdots, l_{1}$ are fixed integers and $l_{0}$ takes $r$ different values in the set $\{0,1, \ldots, r-1\}$. Let $\varphi(j)=0, a_{1} \cdots a_{\nu}$, where

$$
\begin{gathered}
a_{1}=l_{0}+\sum_{h=1}^{\nu-1} e_{h} v_{h+1,1}(\bmod r) \\
a_{2}=l_{1}+\sum_{h=2}^{\nu-1} e_{h} v_{h+1,2}(\bmod r) \\
----------- \\
a_{\nu-1}=l_{\nu-2}+l_{\nu-1} v_{\nu, \nu-1}(\bmod r) \\
a_{\nu}=1_{\nu-1} .
\end{gathered}
$$

It is obvious that and $a_{1}$ takes $r$ different values in the set $\{0,1, \ldots, r-1\}$.

From the Lemma 2.1 we have 


$$
\begin{aligned}
\varphi(m+j) & =\left(0, g_{1}^{m} g_{2}^{m} \cdots g_{\nu}^{m} g_{\nu+1}^{m} \cdots g_{n+\nu}^{m}\right) *\left(0, a_{1} a_{2} \cdots a_{\nu}\right) \\
& =0, b_{1} b_{2} \cdots b_{\nu} g_{\nu+1}^{m} \cdots g_{n+\nu}^{m}
\end{aligned}
$$

where

$$
\begin{gathered}
b_{\nu}=g_{\nu}^{m}+a_{\nu}(\bmod r) \\
b_{\nu-1}=g_{\nu-1}^{m}+a_{\nu-1}(\bmod r) \\
----------- \\
b_{2}=g_{2}^{m}+a_{2}(\bmod r) \\
b_{1}=g_{1}^{m}+a_{1}(\bmod r)
\end{gathered}
$$

Since $0 \leq l^{\prime}<r^{\nu-1}$, we shall search $l^{\prime}$ in the form $l^{\prime}=\left(l^{\prime}{ }_{\nu-1} \cdots l_{1}{ }^{\prime}\right)_{r}$, where $l_{1}{ }^{\prime}, \cdots, l^{\prime}{ }_{\nu-1}$ are unknown quantities. Then $l^{\prime} r=\left(l^{\prime}{ }_{\nu-1} \cdots l_{1}{ }^{\prime} 0\right)_{r}$. When $l^{\prime} r \leq i<(l+1) r$ then $i=\left(l_{\nu-1}{ }_{\nu-l_{1}}{ }^{\prime}\right.$ $\left.l_{0}\right)_{r}$, for $0 \leq l_{0}<r$.

Let us denote $\varphi(i)=0, c_{1} c_{2} \cdots c_{\nu}$ where

$$
\begin{gathered}
c_{1}=l_{0}{ }^{\prime}+\sum_{h=1}^{\nu-1} l_{h}{ }^{\prime} v_{h+1,1}(\bmod r) \\
c_{2}=l_{1}{ }^{\prime}+\sum_{h=2}^{\nu-1} l_{h}{ }^{\prime} v_{h+1,2}(\bmod r) \\
c_{\nu-1}=l_{\nu-2}^{\prime}+l_{\nu-1}^{\prime} v_{\nu, \nu-1}(\bmod r) \\
c_{\nu}=l_{\nu-1} .
\end{gathered}
$$

Then we have

$$
\begin{aligned}
& \begin{array}{lll}
\delta_{1} & \delta_{2} & \delta_{\nu-1}
\end{array} \\
& \varphi(m)+\varphi(i)(\bmod 1)=0, g_{1}^{m} g_{2}^{m} \cdots g_{\nu-1}^{m} g_{\nu}^{m} g_{\nu+1}^{m} \cdots g_{\nu+n}^{m} \\
& +0, c_{1} c_{2} \cdots c_{\nu-1} c_{\nu} \\
& 0, d_{1} d_{2} \cdots d_{\nu-1} d_{\nu} g_{\nu+1}^{m} \cdots g_{\nu+n}^{m}
\end{aligned}
$$

where $\delta_{1}, \delta_{2}, \cdots, \delta_{\nu-1}$ are the step-by-step carries and else

$$
\begin{gathered}
d_{\nu}=g_{\nu}^{m}+c_{\nu}(\bmod r) \\
d_{\nu-1}=g_{\nu-1}^{m}+\delta_{\nu-1}+c_{\nu-1}(\bmod r) \\
------ \\
d_{2}=g_{2}^{m}+\delta_{2}+c_{2}(\bmod r) \\
d_{1}=g_{1}^{m}+\delta_{1}+c_{1}(\bmod r)
\end{gathered}
$$

For the demonstration of the equality (2.16) we make equal the numbers, constructed in (2.17) and (2.18), and we get

$$
\begin{aligned}
& l_{\nu-1}^{\prime} \equiv l_{\nu-1}(\bmod r) \\
& l_{\nu-2}^{\prime}+l_{\nu-1}^{\prime} v_{\nu, \nu-1}+\delta_{\nu-1} \equiv l_{\nu-2}+l_{\nu-1} v_{\nu, \nu-1}(\bmod r) \\
& \text { - - - - - - - - - - - - - - - - - - } \\
& l_{1}^{\prime}+\sum_{h=2}^{\nu-1} l_{h}^{\prime} v_{h+1,2}+\delta_{2} \equiv l_{1}+\sum_{h=2}^{\nu-1} l_{h} v_{h+1,2}(\bmod r) \\
& l_{0}^{\prime}+\sum_{h=1}^{\nu-1} l_{h}^{\prime} v_{h+1,1}+\delta_{1} \equiv l_{0}+\sum_{h=1}^{\nu-1} l_{h} v_{h+1,1}(\bmod r) \text {. }
\end{aligned}
$$

Since $0 \leq l_{\nu-1}, l^{\prime}{ }_{\nu-1}<r$, then equation $l_{\nu-1} \equiv l_{\nu-1}(\bmod r)$ has the only solution $l_{\nu-1}=l_{\nu-1}$. Consecutively we solve the left over equations and get uniquely integer number $l^{\prime}=\left(l_{\nu-1}{ }_{\nu-1} \cdot l_{1}{ }^{\prime}\right)_{r}$, such that $0 \leq l^{\prime}<r^{\nu-1}$.

Since $l_{0}$ takes $r$ different values in the set $\{0,1, \ldots, r-1\}$, then and $l_{0}{ }^{\prime}$ takes $r$ different values in the set $\{0,1, \ldots, r-1\}$ and $l^{\prime} r \leq i<(l+1) r$.

Finally, we establish a bijection between the sets from the two sides of the equation (2.16).

Let $p$ and $q$ be such that $0 \leq p, q<r^{s}, p \neq q$ and $p^{\prime}$ and $q^{\prime}$ are the numbers, satisfying the equality (2.16). We shall prove that $p^{\prime} \neq q^{\prime}$. Let us admit that $p^{\prime}=q^{\prime}=\alpha$. Then we have

$$
\begin{aligned}
\{\varphi(m+j): m & \left.\equiv 0\left(\bmod r^{\nu}\right), p r \leq j<(p+1) r\right\} \\
& =\left\{\varphi(m)+\varphi(i)(\bmod 1): m \equiv 0\left(\bmod r^{\nu}\right), \alpha r \leq i<(\alpha+1) r\right\} .
\end{aligned}
$$


and

$$
\begin{aligned}
\{\varphi(m+j): m & \left.\equiv 0\left(\bmod r^{\nu}\right), q r \leq j<(q+1) r\right\} \\
& =\left\{\varphi(m)+\varphi(i)(\bmod 1): m \equiv 0\left(\bmod r^{\nu}\right), \alpha r \leq i<(\alpha+1) r\right\}
\end{aligned}
$$

Then we have

$$
\begin{aligned}
\{\varphi(m+j): m & \left.\equiv 0\left(\bmod r^{\nu}\right), p r \leq j<(p+1) r\right\} \\
& =\left\{\varphi(m+j): m \equiv 0\left(\bmod r^{\nu}\right), q r \leq j<(q+1) r\right\} .
\end{aligned}
$$

This is a contradiction, since the function $\varphi$ is an injection; so the equation (2.16) is proved.

From (2.15) and (2.16) we get

$$
\begin{gathered}
\left\{\varphi(m+j): m \equiv 0\left(\bmod r^{\nu}\right), 0 \leq j<r^{\nu}\right\}=\bigcup_{l^{\prime}=0}^{r^{\nu-1}-1}\{\varphi(m)+\varphi(i)(\bmod 1): m \equiv 0 \\
\left.\left(\bmod r^{\nu}\right), l^{\prime} r \leq i<\left(l^{\prime}+1\right) r\right\}=\left\{\varphi(m)+\varphi(j)(\bmod 1): m \equiv 0\left(\bmod r^{\nu}\right), 0 \leq j<r^{\nu}\right\} .
\end{gathered}
$$

The lemma is proved.

3. AN ESTIMATION FROM ABOVE FOR THE DIAPHONY OF $\mathbf{P}_{\mathrm{r}}$-SEQUENCES.

THEOREM 3.1. Let in the guiding matrix $\left(v_{s, j}\right)$ every $v_{s, s}=1$ and for $j>s$ every $v_{s, j}=0$ and let $\sigma=(\varphi(i))_{\imath=0}^{\infty}$ be the $P_{r}$-sequence which is produced by the $\left(v_{s, j}\right)$. Then for every positive integer $N$ we have

where the constant $c(r)$ is given by

$$
F_{N}(\sigma) \leq c(r) N^{-1}(\log ((r-1) N+1))^{1 / 2},
$$

$$
c(r)=\pi\left(\left(r^{2}-1\right) / 3 \log r\right)^{1 / 2} .
$$

The proof of this theorem is based on a non-trivial estimate for the trigonometric sum of an arbitrary $P_{r}$ - sequence.

\subsection{AN ESTIMATION OF THE TRIGONOMETRIC SUM OF ARBITRARY $P_{r}-S E Q U E N C E$.}

Let $X=\left(x_{n}\right)_{n=0}^{\infty}$ is arbitrary sequence in interval $E . A$ trigonometric sum, $S_{N}(X: h)$, of the sequence $X$, where $h$ is an integer is the quantity

$$
S_{N}(X ; h)=\sum_{n=0}^{N-1} \exp \left(2 \pi i h x_{n}\right) .
$$

LEMMA 3.1. Let $N=P+Q$, where $P$ and $Q$ are arbitrary integers. Then for every integer $h$ and arbitrary sequence $X=\left(x_{n}\right)_{n=0}^{\infty}$ we have

where

$$
\left|S_{N}(X ; h)\right| \leq\left|S_{P}(X ; h)\right|+\left|S_{P}^{Q}(X ; h)\right| \text {, }
$$

The proof of lemma is obvious

$$
S_{P}^{Q}(X ; h)=\sum_{n=P}^{P+Q-1} \exp \left(2 \pi i x_{n}\right)
$$

LEMMA 3.2. Let $N=a r^{n}$, where $a \geq 1$ and $n \geq 0$ are integers.

Then for every integer $h$ we have

$$
\left|S_{N}(X ; h)\right| \leq \sum_{\imath=1}^{a}\left|S_{(\imath-1) r^{n}}^{r^{n}}(X ; h)\right|
$$

The proof of lemma is based of Lemma 3.1 and is done by induction on $a$.

Let $a$ be an arbitrary integer and $q$ a positive integer. We define the function $\delta_{q}(a)$ by

$$
\delta_{q}(a)=\left\{\begin{array}{l}
1, \text { if } a \equiv 0(\bmod q) \\
0, \text { if } a \neq 0(\bmod q)
\end{array}\right.
$$

It is well known that for every integer $a$ and every natural $q$ we have

$$
\sum_{x=0}^{q-1} \exp (2 \pi i a x / q)=q_{q}^{\delta}(a)
$$

LEMMA 3.3. Let $N \geq 1$ be an integer and

$$
N=\sum_{\jmath=0}^{\infty} a_{\jmath} r^{\nu}, a_{\jmath} \in\{0,1, \cdots, r-1\}(j=0,1, \cdots)
$$

be its $r$-adic representation.

Let in the guiding matrix $\left(v_{s, j}\right)$ every $v_{s, s}=1$ and for $j>s$ every $v_{s, j}=0$ and $\sigma=(\varphi(n))_{n=0}^{\infty}$ be the $P_{r}$-sequence which is product of $\left(v_{s, j}\right)$.

Then for every integer $h$ we have

$$
\left|S_{N}(\sigma ; h)\right| \leq \sum_{\jmath=0}^{\infty} a_{\jmath} r^{\nu} \delta_{r}(h)
$$

PROOF. Let $N \geq 1$ be an integer with $r$-adic representation of a type (3.2). 
We shall prove that for every integer $h$ and for every sequence $X$ in interval $E$ we have the estimation

$$
\left|S_{N}(X ; h)\right| \leq \sum_{\jmath=0}^{\infty} \sum_{m=1}^{a_{j}}\left|S_{(m-1) r}^{r}(X ; h)\right|,
$$

where we have the supposition that when $a_{\jmath}=0$, the inside sum is 0 .

Let $h$ be an integer. For every $N \geq 1$ exists an integer $n$, such that $N<r^{n}$. We shall prove the lemma by the induction on $n$

If $n=1$, then the estimation (3.3) is trivial.

We suppose, that (3.2) is true for every integer $N, 1 \leq N<r^{n}$, where $n$ is some integer.

Let now $N$ such that $r^{n} \leq N<r^{n+1}$. By here we have, that in (3.2) $a_{\jmath}=0$ for $j>n$ Let $N=P+Q$ where $P=a_{n} r^{n}$ and $Q=\sum_{\jmath=0}^{n-1} a, r$.

By Lemma 3.1, Lemma 3.2 and the induction supposition we get

$$
\begin{aligned}
& \left|S_{N}(X ; h)\right| \leq\left|S_{a_{n} r^{n}}(X ; h)\right|+\left|S_{P}^{Q}(X ; h)\right| \leq \sum_{m=1}^{a_{n}}\left|S_{(m-1) r^{n}}^{r^{n}}(X ; h)\right| \\
& +\sum_{\jmath=0}^{n-1} \sum_{m=1}^{a,}\left|S_{(m-1) r}^{r^{\nu}}(X ; h)\right|=\sum_{\jmath=0}^{n} \sum_{m=1}^{a,}\left|S_{(m-1) r}^{r^{\nu}}(X ; h)\right| \\
& =\sum_{\jmath=0}^{\infty} \sum_{m=1}^{a,}\left|S_{(m-1) r}^{r},(X ; h)\right| \text {, }
\end{aligned}
$$

such that (3.3) is proved If $Q=0$, then (3 3) is got by Lemma 3.2.

Let now $j, 0 \leq j \leq n$ be an arbitrary fixed number and consider that $1 \leq m \leq a_{\jmath}$. If $m=1$, then by Lemma 2.2 for the trigonometric sum $S_{0}^{\nu}(\sigma ; h)$ we have

$$
S_{0}^{\nu}(\sigma ; h)=r^{\nu} \delta_{r},(h)
$$

Let now $2 \leq m \leq a_{\jmath}$. Then for the trigonometric sum $S_{(m-1) r}^{r}(\sigma ; h)$, by Lemma 2.4, we have

$$
\begin{gathered}
S_{(m-1) r^{\prime}}(\sigma ; h)=\sum_{n=(m-1) r}^{m r^{\nu}-1} \exp (2 \pi i h \varphi(n))=\sum_{k=0}^{\nu^{\nu}-1} \exp \left(2 \pi i h \left(\varphi\left((m-1) r^{\nu}\right)\right.\right. \\
+\varphi(k)))=\exp \left(2 \pi i h \varphi\left((m-1) r^{\nu}\right)\right) \sum_{k=0}^{r^{\nu}-1} \exp (2 \pi i h \varphi(k)) .
\end{gathered}
$$

Thus for the module of the trigonometric sum $S_{(m-1) r}^{\nu^{\prime}}(\sigma ; h)$ we get

$$
\left|S_{(m-1) r}^{r^{\prime}}(\sigma ; h)\right|=r^{\nu} \delta_{r^{\prime}}(h) \text {. }
$$

From (3.3), (3.4) and (3.5) we get

$$
\left|S_{N}(\sigma ; h)\right| \leq \sum_{\jmath=0}^{\infty} a_{\jmath} r^{\nu} \delta_{r}(h)
$$

The lemma is proved.

\subsection{PROOF OF THEOREM 3.1.}

Let $\left(v_{s, j}\right)$ is an arbitrary guiding matrix, such that on principal diagonal there stand ones, and over him zeros and $\sigma=(\varphi(n))_{n=0}^{\infty}$ is $P_{r}$-sequence, which is bred by the matrix $\left(v_{s, j}\right)$.

We choose $N \geq 1$ arbitrary integer and let has $r$-adic representation in the form

$$
N=\sum_{\jmath=1}^{k} a_{\jmath} r^{n,}\left(a_{j} \in\{1, \cdots, r-1\}, j=1,2, \cdots, k\right),
$$

where

$$
0 \leq n_{1}<n_{2}<\cdots<n_{k} .
$$

are integer numbers.

From Lemma 3.3 for every integer $h$ we have

$$
\left|S_{N}(\sigma ; h)\right| \leq \sum_{j=1}^{k} a_{j} r^{n,} \delta_{r^{n,}}(h) \leq(r-1) \sum_{\jmath=1}^{k} r^{n,} \delta_{r^{n},}(h) .
$$

By the last inequality for the diaphony $F_{N}(\sigma)$ of $\sigma$ we have

$$
\begin{gathered}
\left.\left(N F_{N}(\sigma)\right)^{2}=2 \sum_{h=1}^{\infty} h^{-2} \mid S_{N} \sigma ; h\right)^{2} \leq \\
2(r-1)^{2} \sum_{h=1}^{\infty} h^{-2} \sum_{\jmath=1}^{k} \sum_{\nu=1}^{k} r^{n_{\jmath}+n_{\nu}} \delta_{r^{n}}(h) \delta_{r_{\nu}}(h) \\
=2(r-1)^{2} \sum_{\jmath=1}^{k} \sum_{\nu=1}^{k} r^{n_{\jmath}+n_{\nu}} \sum_{h=1}^{\infty} h^{-2} \delta_{r^{n}}(h) \delta_{r^{n_{\nu}}}(h) .
\end{gathered}
$$

If the matrix ||$a_{\jmath, \nu}||(1 \leq j, \nu \leq k)$ is symmetric then we have

$$
\sum_{\jmath=1}^{k} \sum_{\nu=1}^{k} a_{j, \nu}=2 \sum_{j=1}^{k} \sum_{\nu=1}^{\jmath} a_{\jmath, \nu}-\sum_{j=1}^{k} a_{\jmath, \jmath} .
$$

By here and (3.7) we get

$$
\begin{gathered}
\left(N F_{N}(\sigma)\right)^{2} \leq 4(r-1)^{2} \sum_{\jmath=1}^{k} \sum_{\nu=1}^{J} r^{n_{\jmath}+n_{\nu}} \sum_{h=1}^{\infty} h^{-2} \delta_{r^{n_{\jmath}}}(h) \delta_{r^{n_{\nu}}}(h) \\
-2(r-1)^{2} \sum_{\jmath=1}^{k} r^{2 n_{j}} \sum_{h=1}^{\infty} h^{-2} \delta_{r^{n_{\jmath}}}(h) .
\end{gathered}
$$

For $j$ and $\nu$ such that $1 \leq \nu \leq j \leq k$, we have

$$
\delta_{r^{n_{j}}}(h) \delta_{r^{n_{\nu}}}(h)=\delta_{r^{n^{\prime}}}(h),
$$


for every integer $h$.

Beside this we have

$$
\sum_{h=1}^{\infty} h^{-2} \delta_{r^{n}}(h)=\pi^{2} / 6 r^{2 n} .
$$

By (3.8), (3.9) and (3.10) we have

$$
\left(N F_{N}(\sigma)\right)^{2} \leq\left(2 \pi^{2}(r-1)^{2} / 3\right) \sum_{\jmath=1}^{k} \sum_{\nu=1}^{\jmath} r^{n_{\nu}-n,}-\left(\pi^{2} / 3\right)(r-1)^{2} k
$$

For the sum in last equality holds, that

$$
\sum_{\jmath=1}^{k} \sum_{\nu=1}^{\jmath} r^{n_{\nu}-n_{\jmath}}=\sum_{\nu=1}^{k} r^{n_{\nu}} \sum_{\jmath=\nu}^{k} r^{-n_{\jmath}}<\sum_{\nu=1}^{k} r^{n_{\jmath}} \sum_{n=n_{\nu}}^{\infty} r^{-n}=(r k) /(r-1)
$$

From (3.11) and (3.12) we have

$$
\left(N F_{N}(\sigma)\right)^{2} \leq\left(\pi^{2} / 3\right)\left(r^{2}-1\right) k
$$

From (3.6) we get that

$$
N \geq \sum_{\jmath=1}^{k} r^{n_{\jmath}} \geq \sum_{\jmath=0}^{k-1} r^{\nu}=\left(r^{k}-1\right) /(r-1)
$$

Thus we discover

$$
k \leq(\log ((r-1) N+1)) / \log r
$$

From (3.13) and (3.14) we have

$$
F_{N}(\sigma) \leq \pi\left(\left(r^{2}-1\right) / 3 \log r\right)^{1 / 2} N^{-1}(\log ((r-1) N+1))^{1 / 2}
$$

The Theorem 3.1 is proved.

In the case, where the guiding matrix $\left(v_{s, j}\right)$ is a unit matrix $I$, the sequence which is bred by $I$ is called Van der Corput-Halton's sequence. In 1935 it was first introduced by Van der Corput [18] and generalized in 1960 by Halton [19].

In this case the operation * turns out to be a simple addition.

By $\varphi_{r}(i)(i=0,1, \cdots)$ we signify the general term of the Van der Corput-Halton-sequence.

For $r=2$ the sequence of general terms $\varphi_{2}(i)(i=0,1, \cdots)$ is called Van der Corput-sequence.

By Theorem 3.1 we can get the following corollaries.

COROLLARY 3.1. Let $\sigma=\left(\varphi_{r}(i)\right)_{\imath=0}^{\infty}$ be the Van der Corput-Halton-sequence. Then for every positive integer $N$, we have

$$
F_{N}(\sigma) \leq c(r) N^{-1}(\log ((r-1) N+1))^{1 / 2},
$$

where the constant $c(r)$ is determined by the equality (3.1).

COROLLARY 3.2. Let $\sigma=(\varphi(i))_{i=0}^{\infty}$ be the Van der Corput-sequence. Then for every $N \geq 1$ we have

$$
F_{N}(\sigma)<4 N^{-1}(\log N)^{1 / 2} .
$$

COROLLARY 3.3. Let $\sigma=(\varphi(i))_{\imath=0}^{\infty}$ be arbitrary binary $P_{r}$-sequence. Then

$$
\overline{\lim }_{N \rightarrow \infty}\left(N F_{N}(\sigma)\right) /(\log N)^{1 / 2} \leq \pi /(\log 2)^{1 / 2}=3,7773 \cdots
$$

We note that the Corollary 3.1 and Corollary 3.2 are announced without proof by Proinov and Grozdanov [20] and proved by Proinov and Grozdanov [21].

\section{ON QUADRATIC DISCREPANCY OF THE SYMMETRIC SEQUENCE PRODUCED BY THE ARBITRARY $P_{\mathrm{r}}$-SEQUENCE.}

In this section, we given an application of Theorem 3.1 to the problem of finding infinite sequences in $E$, with the best possible order of magnitude for the quadratic discrepancy.

We need the notion of symmetric sequence (see [11]). A sequence $\sigma=\left(x_{n}\right)_{n=0}^{\infty}$ in $E$ is called symmetric of for every integer $n \geq 0$ we have $x_{2 n}+x_{2 n+1}=1$. A symmetric sequence $\widetilde{\sigma}=\left(b_{n}\right)_{n=0}^{\infty}$ in $E$ is said to be produced by an infinite sequence $\sigma=\left(a_{n}\right)_{n=0}^{\infty}$, if for every integer $n \geq 0$ we either have $a_{n}=b_{2 n}$ or $a_{n}=b_{2 n+1}$. Obviously, every infinite sequence in $E$ produce at least one symmetric sequence.

By Sobol ([5], p. 117) is clear that the exact order of quadratic discrepancy of $P_{2}$-sequence is $O\left(N^{-1} \log N\right)$.

We shall prove that the quadratic discrepancy of arbitrary symmetric sequence, which is produced by arbitrary $P_{r}$-sequence has exact order $O\left(N^{-1}(\log N)^{1 / 2}\right)$. In the foundation of this problem stands Theorem A, proved by Proinov and Grozdanov [20]. 
By this and Theorem 31 follows

THEOREM 41 Let $\widetilde{\sigma}$ be an arbitrary symmetric sequence in $E$, which is produced by an arbitrary $P_{r}$-sequence Then for every integer $N \geq 2$ we have

$$
\left.T_{N}(\widetilde{\sigma})<c(r) N^{-1}(\log (r-1) N)\right)^{1 / 2}+N^{-1},
$$

where $c(r)$ is defined by the equality $(31)$

From Theorem 41 for the case $r=2$ we have

$$
\overline{\lim }_{N \rightarrow \infty} N T_{N}(\widetilde{\sigma}) /(\log N)^{1 / 2} \leq 1 /(\log 2)^{1 / 2}=1,201 \cdots,
$$

for every symmetric sequence $\widetilde{\sigma}$ produced by the $P_{2}$-sequence

We note that Faure [22] proved that for the symmetric sequence $\widetilde{\sigma}$, produced by the Van der Corput-sequence, the constant $\overline{\lim }_{N \rightarrow \infty}\left(N T_{N}(\widetilde{\sigma}) /(\log N)^{1 / 2}\right)$ is between 0,298 and 0,321

\section{REFERENCES}

1. WEYL, H, Uber die gleichverteilung von zahlen mod. eins, Mat. Ann. 77 (1916), 313-352

2. ROTH, K.F., On irregularities of distribution, Mathematika 1 (1954), 73-79.

3. ZINTERHOF, P., Uber einige abschatzungen bei der bei der approximation von funktionen mit gleichverteilungsmethoden, Sitzungsber.Osterr.Akad.Wiss. Math-Naturwiss.Kl 185 (1976), 121-132.

4. ZINTERHOF, P. \& STEGBUCHNER, H., Trigonometrische approximation mit gleichverteilungsmethoden, Studia Sci Math.Hungar 13 (1978), 273-289.

5 SOBOL, I M , Multidimentional Quadrature Formulas and Haar Function.Izdat "Nauka", Moscow, 1969

6. KUIPERS, L., Simple proof of a theorem of J K Koksma, Nieuw Tijdsch.Wisk. 55 (1967), 108-111

7. NEIDERREITER, H., Application of diophantine approximation to numerical integration, in Diophantine Approximation and its Application, C.F. Osgoot, Ed., 129-199.

8. PROINOV, PD., On irregularities of distribution, C.R. Acad. Bulgar Sci. 39, No 9 (1986), 31-34.

9. PROINOV, P.D., Points of constant type and upper bound for the meam-square discrepancy of a class of sequences, C R Acad. Bulgar Sci. 35 (1982), 753-755.

10. PROINOV, P.D, Estimation of $L^{2}$ discrepancy of a class of infinite sequences, C.R. Bulgar Sci. 36 (1983), 37-40.

11. PROINOV,P.D., On the $L^{2}$ discrepancy of some infinite sequences, Serdica 11 (1985), 3-12.

12. SOBOL, I.M., On the distribution of points in a cube and approximate calculation of integrals, J. Approx. Math \& Math Phys. 7, No. 4 (1967), 784-802.

13. SOBOL, I.M., A point uniform distribution in multidimentional cube, Izdat "Znani", Moscow 1985

14. FAURE, H., Discrepances de suites associees a un systeme de numeration (en dimention un), Bull. Soc. Math. France 109 (1981), 143-182.

15. FAURE, H., Discrepance de suites associees a un systeme de numeration (en dimention s), Acta Arith., XII (1982), 337-351.

16. NEIDERREITER, $H$., Point sets and sequences with small discrepancy, Mh. Math. 104 (1987), 273-337.

17. NEIDERREITER, $\mathbf{H}$, Low-discrepancy and low-dispersion sequences, J. Number Theory $\mathbf{3 0}$ (1988), 51-70.

18. VAN DER CORPUT, J G, Verteilundsfunktionen, Proc Kon.Ned. Akad. Wetensch. 38 (1935), 813-821.

19. HALTON, J.H., On the efficiency of certain quasi-random sequences of points in evaluating multi-dimensional integrals, Numer Math. 2 (1960), 84-90.

20. PROINOV, P.D \& GROZDANOV, V ST, Symmetrization of the Van der Corput-Halton sequence, C.R Acad. Bulgar Sci. 40, No. 8 (1987), 5-8.

21. PROINOV, P.D. \& GROZDANOV, V ST., On the diaphony of the Van der Corput-Halton sequence, J. Number Theory 30 (1988), 94-104

22. FAURE, H., Discrepance quadratique de suite de Van der Corput et sa symetrique, Acta Arith., LV (1990), 333-350. 


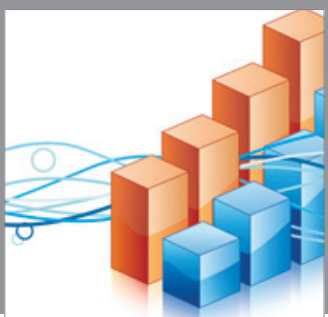

Advances in

Operations Research

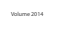

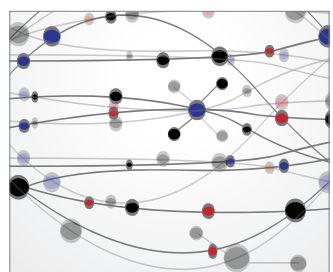

\section{The Scientific} World Journal
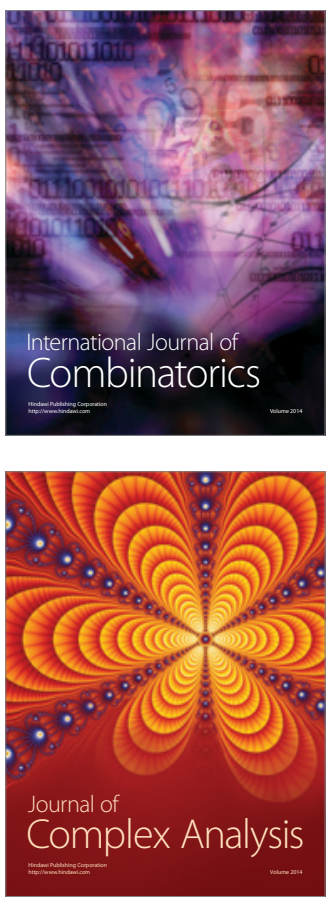

International Journal of

Mathematics and

Mathematical

Sciences
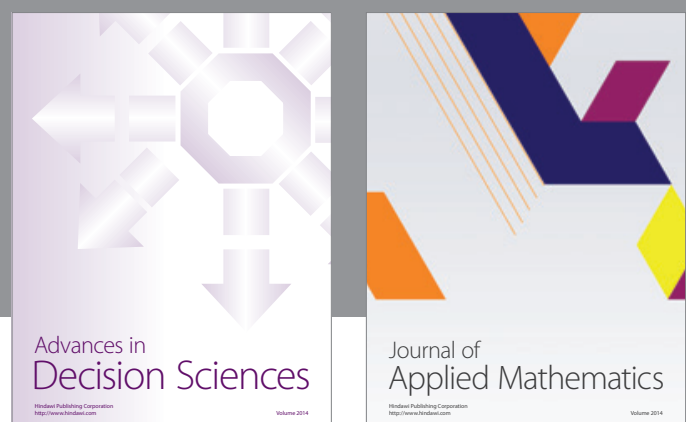

Journal of

Applied Mathematics
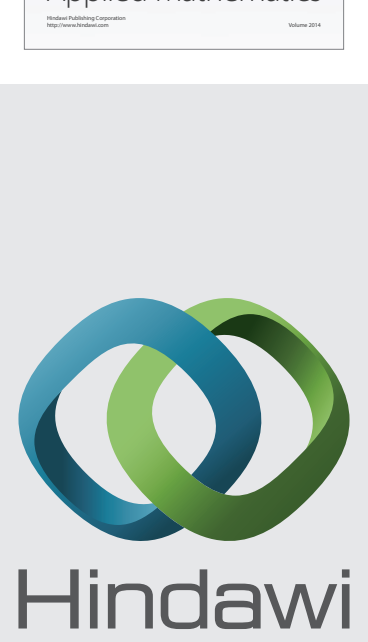

Submit your manuscripts at http://www.hindawi.com
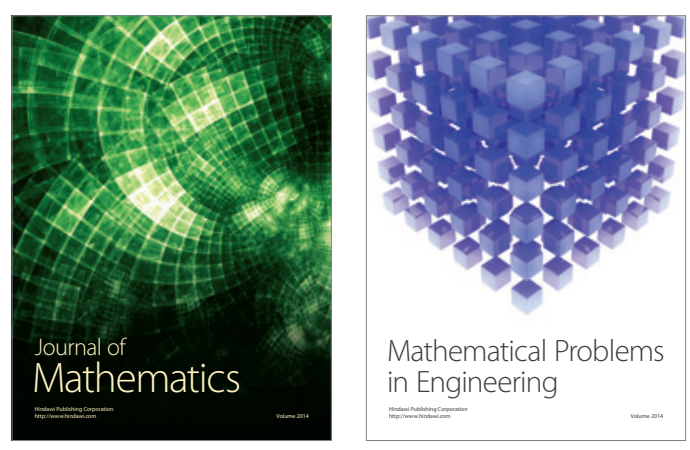

Mathematical Problems in Engineering
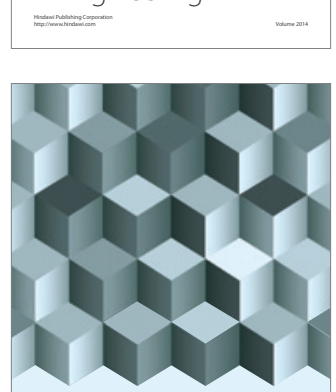

Journal of

Function Spaces
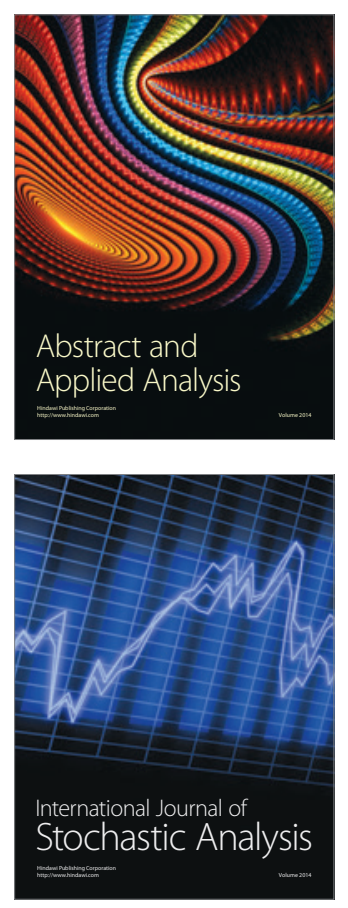

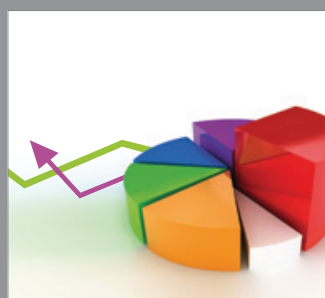

ournal of

Probability and Statistics

Promensencen
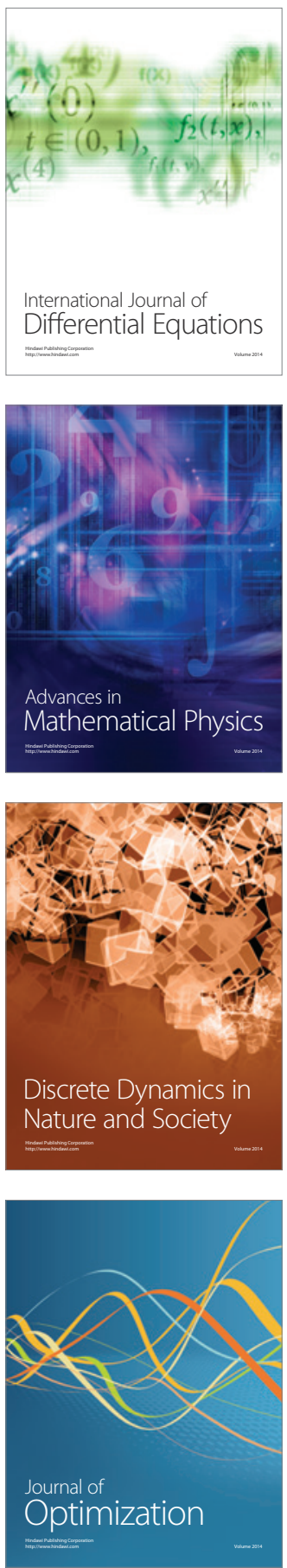